\title{
THE PARTITION PROPERTY FOR CERTAIN EXTENDIBLE MEASURES ON SUPERCOMPACT CARDINALS
}

\author{
DONALD H. PELLETIER ${ }^{1}$
}

\begin{abstract}
We give an alternate characterization of a combinatorial property of measures on $p_{k} \lambda$ introduced by Menas. We use this characterization to prove that if $\kappa$ is supercompact, then all measures on $p_{\kappa} \lambda$ in a certain class have the partition property. This result is applied to obtain a self-contained proof that if $\alpha$ is supercompact and $\lambda$ is the least measurable cardinal greater than $\alpha$, then Solovay's "glue-together" measures on $p_{k} \lambda$ are not $2^{\lambda}$-extendible.
\end{abstract}

0. Introduction. The well-established study of combinatorial properties of measures on a measurable cardinal $\kappa$ was initially extended to the context of measures on $p_{\kappa} \lambda$ by Jech [2]. Among these combinatorial properties, one of the most interesting is the so-called partition property. Rowbottom [9] proved that every normal measure on a measurable cardinal has the partition property. This is the key step in Rowbottom's proof of the fact that every measurable cardinal is Ramsey. The natural analogue of the partition property for normal measures on $p_{\kappa} \lambda$ has turned out to be even more interesting because, unlike in the case for normal measures on a measurable cardinal, it does not hold universally. The following results are known.

THeOREM A (MENAS [5]). If $\kappa$ is supercompact and $\lambda$ is a cardinal $>\kappa$, then there exist $2^{2^{\lambda<\kappa}}$-many normal measures on $p_{\kappa} \lambda$ with the partition property.

TheOREM B (Solovay; PUblished IN Menas [5]). If $\kappa$ is supercompact and $\lambda>\kappa$ is $\beta$-supercompact for some $\beta>\lambda$, then there is a normal measure on $p_{\kappa} \beta$ without the partition property.

Theorem C (Solovay, assuming GCH; Menas, without GCH [5]). If $\kappa$ is supercompact, then for certain small cardinals $\left(e . g . \lambda=\kappa^{+}\right.$or $\left.\lambda=2^{\kappa}\right)$ every normal measure on $p_{\kappa} \lambda$ has the partition property.

The principal result of this paper is Theorem 3 in $\$ 3$; it is in the spirit of Theorem $\mathbf{C}$ and it asserts that if $\kappa$ is supercompact, then the partition property holds for all normal measures in a fairly extensive class.

Received by the editors October 19, 1979; presented at the Union College Set Theory Conference in May, 1979 and announced in [6] and [7].

AMS (MOS) subject classifications (1970). Primary 02K35, 04 A20.

Key words and phrases. Supercompact cardinals, measures with the partition property, extendible measures, "glue-together" measures, restriction measures.

${ }^{1}$ This work was partially supported by the National Science and Engineering Research Council of Canada under Grant \# A8216. 
$\$ 1$ introduces notation, defines the various combinatorial properties to be studied, and recalls some elementary facts.

$\$ 2$ contains a theorem which gives an alternate characterization of a combinatorial property used by Menas in his proofs of Theorems $\mathbf{A}$ and $\mathbf{C}$.

$\$ 3$ depends on $\$ 2$ and contains the statement and proof of the main result; it concludes with an application.

We wish to thank Smith College for generously providing hospitality, office space, and secretarial services during 1978-79 when research for this paper was completed. We owe a special debt to Stan Wagon and Jim Henle for shooting down many high-flying conjectures, often with laser-beam speed and accuracy.

1. Notation, definitions, and elementary facts. Our notation is adopted so as to agree, by and large, with that of Menas [5].

$\alpha, \beta, \gamma, \xi$ denote infinite ordinals which may or may not also be cardinals.

$\delta, \kappa, \lambda$ denote infinite cardinals and we always assume that $\kappa$ is regular and that $\delta, \lambda>\kappa>\omega$.

$\mu, \nu, \tau$ denote measures.

$\phi, \psi, \chi$ denote formulas in $L_{\mathrm{ZF}}$, the language of $\mathrm{ZF}$.

$p_{\kappa} \lambda$ denotes the set of all those subsets of $\lambda$ which have cardinality strictly less than $\kappa$.

We write $f: A \rightarrow B$ to abbreviate the assertion that $f$ is a function with domain $A$ and range included in $B . P(A)$ denotes the power set of $A$.

A measure on a set $A$ is a function $\mu: P(A) \rightarrow\{0,1\}$ which satisfies the following:

(i) $\mu(\{a\})=0$ for every $a \in A$;

(ii) $\mu(A)=1$; and

(iii) $\mu(X)=1$ iff $\mu(A-X)=0$ for every $X \in P(A)$.

A measure $\mu: P(A) \rightarrow\{0,1\}$ is called $\kappa$-additive if for every $\beta<\kappa$ and for every pairwise disjoint collection $\left\{X_{\alpha} \mid \alpha<\beta\right\} \subseteq P(A), \mu\left(\cup_{\alpha<\beta} X_{\alpha}\right)=\Sigma_{\alpha<\beta} \mu\left(X_{\alpha}\right)$.

If $\mu$ is an $\aleph_{1}$-additive measure on a set $A$, then $j_{\mu}: V \rightarrow M_{\mu} \simeq V^{A} / \mu$ will always denote the canonical elementary embedding of the universe into the transitive collapse, $M_{\mu}$, of the ultrapower, $V^{A} / \mu$, of the universe with respect to $\mu$. We will usually abuse language and call $M_{\mu}$ the ultrapower associated with $\mu$. In this context, when $f: A \rightarrow V,{ }^{\top} f^{\urcorner \mu}$ denotes that element of $M_{\mu}$ to which the $\mu$-equivalence class of $f$ is sent by the transitive collapsing map. When $f$ is explicitly defined by $a \mapsto f(a)$, we will write ${ }^{\top} a \mapsto f(a)^{\top \mu}$ instead of ${ }^{\top} f^{\top \mu}$.

A measure $\mu$ on $p_{\kappa} \lambda$ is normal if for all $f: p_{\kappa} \lambda \rightarrow \lambda$, whenever

$$
\mu\left(\left\{P \in p_{\kappa} \lambda \mid f(P) \in P\right\}\right)=1,
$$

there exists some $\alpha<\lambda$ such that $\mu\left(\left\{P \in p_{\kappa} \lambda \mid f(P)=\alpha\right\}\right)=1$.

A measure $\mu$ on $p_{\kappa} \lambda$ is fine if for every $\alpha<\lambda, \mu\left(\left\{P \in p_{\kappa} \lambda \mid \alpha \in P\right\}\right)=1$.

A cardinal $\kappa$ is measurable if there is a $\kappa$-additive measure on $\kappa$.

When $\alpha \geqslant \lambda$, we say $\kappa$ is $\alpha$-supercompact if there is a fine, $\kappa$-additive, normal measure on $p_{\kappa} \alpha$. If $\kappa$ is $\alpha$-supercompact for all $\alpha>\kappa$, we say $\kappa$ is supercompact. 
Henceforth, the word "measure", when it refers to a measure on some $p_{\kappa} \alpha$, will be understood to mean fine, $\kappa$-additive, normal measure; when it refers to a measure on a cardinal $\kappa$, it will be understood to mean $\kappa$-additive measure, and in this case, the additional attribute "normal" will be supplied where appropriate.

For $P \in p_{\kappa} \alpha$, let $\hat{P}=\left\{Q \in p_{\kappa} \alpha \mid P \subseteq Q\right\}$. It is easy to verify, using fineness in conjunction with $\kappa$-additivity, that $\mu(\hat{P})=1$ for every $P \in p_{\kappa} \alpha$ and every measure $\mu$ on $p_{\kappa} \alpha$.

Suppose $\kappa<\alpha<\beta$ and $q: p_{\kappa} \beta \rightarrow p_{\kappa} \alpha$. If $\mu$ is a measure on $p_{\kappa} \beta$, then $q_{*}(\mu)$ is the function on $P\left(p_{k} \alpha\right)$ defined by $q_{*}(\mu)(X)=1$ iff $\mu\left(\left\{P \in p_{k} \beta \mid q(P) \in X\right\}\right)=1$. It is well known that $q_{*}(\mu)$ is a measure on $p_{\kappa} \alpha$ provided that for every $\gamma<\alpha$, $\mu\left(\left\{P \in p_{\kappa} \beta \mid \gamma \in q(P)\right\}\right)=1$. A special case, in which this proviso is satisfied, arises when $q$ is the function $P \mapsto P \cap \alpha$; in this case the measure $q_{*}(\mu)$ is called the restriction of $\mu$ to $p_{\kappa} \alpha$ and will of ten be denoted by $\mu \uparrow p_{\kappa} \alpha$.

We will also want to view this relationship from the other direction. Suppose $\kappa<\alpha<\beta$ and that $\nu$ is a measure on $p_{\kappa} \alpha$. We say that $\nu$ is $\beta$-extendible if there exists a measure $\mu$ on $p_{\kappa} \beta$ such that $\nu=\mu \uparrow p_{\kappa} \alpha$. A measure on $p_{\kappa} \alpha$ is extendible if it is $\beta$-extendible for every $\beta \geqslant \alpha$. This concept was introduced by Magidor who observes that $\nu$ is extendible iff the set $\{\beta>\alpha \mid \nu$ is $\beta$-extendible $\}$ is unbounded. He also proved the following theorem.

THEOREM D (MAGIDOR [3]). If $\kappa$ is supercompact, then for every $\lambda>\kappa$, there exists $\left(2^{\lambda<k}\right)^{+}$-many extendible measures on $p_{\kappa} \lambda$.

For any set $X$, let $[X]^{2}=\{\{P, Q\} \mid P, Q \in X$ and $P \neq Q\}$. A subset $X$ of $p_{\kappa} \lambda$ is called homogeneous for $F$, where $F:\left[p_{\kappa} \lambda\right]^{2} \rightarrow\{0,1\}$, if $F$ is constant on $\{\{P, Q\} \in$ $[X]^{2} \mid P \subset Q$ or $\left.Q \subset P\right\}$. A measure $\mu$ on $p_{\kappa} \lambda$ is said to have the partition property (which we will abbreviate as $\operatorname{Part}(\mu)$ ) if for every $F:\left[p_{\kappa} \lambda\right]^{2} \rightarrow\{0,1\}$ there is a subset $X$ of $p_{\kappa} \lambda$ with $\mu(X)=1$ which is homogeneous for $F$.

This is the natural analogue, for measures on $p_{\kappa} \lambda$, of the familiar partition property for a measure $\mu$ on a measurable cardinal $\kappa:$ if $F:[\kappa]^{2} \rightarrow\{0,1\}$, then for some $X \subset \kappa$ with $\mu(X)=1, F$ is constant on $[X]^{2}$. Rowbottom's theorem alluded to earlier [9, Theorem 4.8] includes the result that every normal measure on $\kappa$ has this property.

Another combinatorial property we shall study is one introduced by Menas in [5]. A measure $\mu$ on $p_{\kappa} \lambda$ is said to have property $\chi$ (which we will abbreviate as $\chi(\mu)$ ) if there exists an $f: \kappa \rightarrow \kappa$ such that

$$
\mu\left(\left\{P \in p_{\kappa} \lambda|f(|P \cap \kappa|)=| P \mid \text { and } \forall \alpha<|P \cap \kappa|, f(\alpha)<|P \cap \kappa|\right\}\right)=1 .
$$

The importance of property $\chi$ is embodied in the following theorem.

TheORem E (Menas [5]). If $\kappa$ is supercompact and $\mu$ is any measure on $p_{\kappa} \lambda$, then $\chi(\mu) \rightarrow \operatorname{Part}(\mu)$.

For a proof, we refer the reader to the conjunction of Lemma 2, Lemma 5, and Proposition 1 of [5].

Throughout the remainder of this paper, we will often make implicit use of the standard techniques and results, developed by Solovay, Reinhardt, and Kanamori 
in [10], involving elementary embeddings. To establish the notation in the reader's mind, we state the following specific case of Los' Theorem.

TheOREM F (Los). Let $\kappa$ be $\lambda$-supercompact and let $\mu$ be a measure on $p_{\kappa} \lambda$. If $\psi\left(v_{1}, \ldots, v_{n}\right)$ is a formula of $L_{Z F}$ and $f_{1}, \ldots, f_{n}$ are functions from $p_{\kappa} \lambda$ into $V$, then $M_{\mu} \vDash \psi\left({ }^{\ulcorner} f_{1}^{\top \mu}, \ldots,{ }^{\top} f_{n}^{\top \mu}\right)$ iff $\mu\left(\left\{P \in p_{\kappa} \lambda \mid \psi\left(f_{1}(P), \ldots, f_{n}(P)\right)\right\}\right)=1$.

2. An equivalent version of property $\chi$. The next theorem gives a characterization of property $\chi$ in terms of the behaviour of the associated elementary embedding.

First, recall that if $\mu$ is a measure on $p_{\kappa} \lambda$ and $f: \kappa \rightarrow \kappa$, then $j_{\mu}(f): j_{\mu}(\kappa) \rightarrow j_{\mu}(\kappa)$ and $j_{\mu}(k)>\lambda \geqslant \kappa$; thus it makes sense to ask whether $j_{\mu}(f)(\kappa)=\lambda$ or not.

THEOREM 1. For any measure $\mu$ on $p_{\kappa} \lambda, \chi(\mu)$ iff there exists an $f: \kappa \rightarrow \kappa$ such that $j_{\mu}(f)(\kappa)=\lambda$.

Proof. It follows from [10, Proposition 3.1] that since $\kappa$ and $\lambda$ are both cardinals we have $\kappa={ }^{\top} P \mapsto|P \cap \kappa|^{\top \mu}$ and $\lambda={ }^{\top} P \mapsto|P|^{\top \mu}$. Using this together with Los' Theorem and the fact that $M_{\mu}$ is a substructure of $V$, we conclude that for any $f$ : $\kappa \rightarrow \kappa, \mu\left(\left\{P \in p_{\kappa} \lambda|f(|P \cap \kappa|)=| P \mid\right\}\right)=1$ iff $j_{\mu}(f)(\kappa)=\lambda$. To finish the proof, we observe next that, because $\kappa$ is the least ordinal moved by $j$, we have $\forall \alpha<\kappa$, $j_{\mu}(f)(\alpha)=j_{\mu}(f)\left(j_{\mu}(\alpha)\right)=j_{\mu}(f(\alpha))=f(\alpha)$; in other words $j_{\mu}(f) \supseteq f$ and in particular $j_{\mu}(f) \uparrow \kappa: \kappa \rightarrow \kappa$. This fact, together with the definition of satisfaction and another application of Los' Theorem, implies that

$$
\mu\left(\left\{P \in p_{\kappa} \lambda|\forall \alpha<| P \cap \kappa|, f(\alpha)<| P \cap \kappa \mid\right\}\right)=1 .
$$

COROLlARY TO THE PROOF OF THEOREM 1. The second clause in the combinatorial definition of $\chi(\mu)$ is redundant, i.e., the fact that

$$
\mu\left(\left\{P \in p_{\kappa} \lambda|\forall \alpha<| P \cap \kappa|, f(\alpha)<| P \cap \kappa \mid\right\}\right)=1
$$

is already a consequence of the given data.

This corollary was observed independently by C. Di Prisco who gives a strictly combinatorial proof of it in [1, Lemma 6].

Despite its simplicity, Theorem 1 is extremely useful. We have studied property $\chi$ in greater detail in [8]. For example, another equivalent form of $\chi(\mu)$ for $\mu$ on $p_{\kappa} \lambda$ is this: $\chi(\mu)$ iff there exists an $X \subset p_{\kappa} \lambda$ with $\mu(X)=1$ satisfying $\forall P, Q \in X$, $|P \cap \kappa|=|Q \cap \kappa| \rightarrow|P|=|Q|$. Theorem 1 can be used to provide easier proofs of Theorems $\mathrm{A}$ and $\mathrm{C}$, and in conjunction with other results can be used to prove that the implication $\chi(\mu) \rightarrow \operatorname{Part}(\mu)$ in Theorem $\mathrm{E}$ is not reversible. The proof of the following remark of Menas gives the simplest illustration of this kind of argument.

REMARK. For every measure $\mu$ on $p_{\kappa} \kappa^{+}, \chi(\mu)$.

Proof. Let $f: \kappa \rightarrow \kappa$ be the function $\alpha \mapsto \alpha^{+}$. Then $M_{\mu} \vDash j_{\mu}(f)(\kappa)=\kappa^{+}$, i.e., $j_{\mu}(f)(\kappa)=\left(\kappa^{+}\right)^{M_{\mu}}$. But the usual closure properties of $M_{\mu}$ (see [10, Proposition 3.2]) guarantee that $\left(\kappa^{+}\right)^{M_{\mu}}=\kappa^{+} \cdot \chi(\mu)$ then follows by Theorem 1 . 
3. The main theorem. Let $\kappa$ be supercompact. The main theorem (Theorem 3) asserts that if $\nu$ is a measure on $p_{\kappa} \lambda$ and if a certain condition relating $\lambda$ to the extendibility of $\nu$ is satisfied, then $\chi(\nu)$ (and hence also Part $(\nu)$ by Theorem E). To isolate this condition and to determine the degree of extendibility required, it is instructive to first state and prove in detail a specific case of the theorem. The general assertion can then be extracted by a careful analysis of the proof. It is irrelevant for Theorem 3 which specific case we choose to give; our choice is therefore motivated by the application that we plan to present in Theorem 4.

THEOREM 2. Let $\lambda$ be the least measurable cardinal $>\kappa$. Let $\nu$ be a measure on $p_{\kappa} \lambda$ which is $2^{\lambda}$-extendible. Then $\chi(\nu)$ holds.

Proof. There is a familiar picture associated with restriction measures. Let $\mu$ be a measure on $p_{\kappa} 2^{\lambda}$ such that $\nu=\mu \uparrow p_{\kappa} \lambda$.

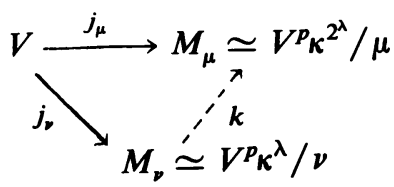

Proposition 3.7 from [10] shows that there is an elementary embedding $k: M_{\nu} \rightarrow M_{\mu}$ which makes the diagram commute. If $q: p_{\kappa} 2^{\lambda} \rightarrow p_{\kappa} \lambda$ is the function $P \mapsto P \cap \lambda$, then the explicit definition of $k$ is as follows: for $h: p_{\kappa} \lambda \rightarrow V, k\left({ }^{\top} h^{\top \nu}\right)={ }^{\top} h \circ q^{\top \mu}$. An additional fact proved about $k$ in [10] is that $k(\kappa)=\kappa$ and $k(\lambda)=\lambda$. Consider the function $f: \kappa \rightarrow \kappa$ defined by

$f(\alpha)=$ the least $\xi$ such that $\alpha<\xi<\kappa$ and $\xi$ is measurable, if such a $\xi$ exists; otherwise, $f(\alpha)=0$.

REMARK. It will in fact be the case that $f(\alpha) \neq 0$; because $\kappa<\lambda, 2^{\kappa} \leqslant 2^{\lambda}$, and since we are assuming that $\kappa$ is $2^{\lambda}$-supercompact, we could invoke [10, Proposition 3.5] which asserts that $2^{\kappa}$-supercompactness of $\kappa$ is already enough to guarantee that $\kappa$ is the $\kappa$ th measurable cardinal. We deliberately define $f$ as above, however, in order to emphasize that no appeal to this last assertion will be necessary.

Continuing with the proof, we have that $M_{\mu} \vDash j_{\mu}(f)(\kappa)=$ the least $\xi$ such that $\kappa<\xi<j_{\mu}(\kappa)$ and $\xi$ is measurable. This is equivalent to saying that $j_{\mu}(f)(\kappa)=$ the least $\xi$ such that $\kappa<\xi<j_{\mu}(\kappa)$ and $M_{\mu} \vDash \xi$ is measurable. Standard facts from [10] imply that $M_{\mu}$ contains all sets whose hereditary cardinality is $<2^{\lambda}$; hence for all $\xi$ in the interval $(\kappa, \lambda], \xi$ is measurable iff $M_{\mu} \vDash \xi$ is measurable. We conclude that $j_{\mu}(f)(\kappa)=\lambda$. From what has been noted about $k$, this can be rewritten as $k\left(j_{\nu}(f)\right)(k(\kappa))=k(\lambda)$, and thus by the elementariness of $k, j_{\nu}(f)(\kappa)=\lambda$. By Theorem 1 , we then get $\chi(\nu)$.

As a corollary to the proof of Theorem 2, we obtain the following generalization. 
TheOREM 3. Let $\kappa$ be supercompact and let $\lambda>\kappa$. Suppose $\phi(\alpha, \xi)$ is a formula in $L_{Z F}$ and that

(i) $\phi\left(\alpha, \xi_{1}\right) \wedge \phi\left(\alpha, \xi_{2}\right) \rightarrow \xi_{1}=\xi_{2}$,

(ii) $\forall \alpha<\kappa, \exists \xi<\kappa \phi(\alpha, \xi)$ and

(iii) for some $\beta>\lambda$, there exists a measure $\mu$ on $p_{\kappa} \beta$ such that $M_{\mu} \vDash \phi(\kappa, \lambda)$.

Then $\chi\left(\mu \uparrow p_{\kappa} \lambda\right)$ (and hence $\mu \uparrow p_{\kappa} \lambda$ has the partition property by Theorem $\mathrm{E}$ ). In particular, if (i) and (ii) hold for $\phi(\alpha, \xi)$ and if $\nu$ on $p_{\kappa} \lambda$ is $\beta$-extendible to a measure $\mu$ on $p_{\kappa} \beta$ for which $M_{\mu} \vDash \phi(\kappa, \lambda)$, then $\chi(\nu)$.

In applications of Theorem 3, the most practical way to verify (iii) is the route taken in the proof of Theorem 2, namely to ascertain that $\phi(\kappa, \lambda)$ holds in the universe and then to choose $\beta$ sufficiently large that the closure properties of $M_{\mu}$ guarantee $\phi(\kappa, \lambda)$ iff $M_{\mu} \vDash \phi(\kappa, \lambda)$.

Theorem 4. Assume that $\kappa$ is supercompact and that $\lambda$ is the least measurable cardinal greater than $\kappa$. Then the "glue-together" measures on $p_{\kappa} \lambda$ are not $2^{\lambda}$-extendible.

Proof. The so-called "glue-together" measures on $p_{\kappa} \lambda$ are defined as follows: fix any normal measure $\tau$ on the measurable cardinal $\lambda$ and fix another measure $\mu$ on $p_{\kappa} \lambda$; for $\alpha<\lambda$, define $q^{\alpha}: p_{\kappa} \lambda \rightarrow p_{\kappa} \alpha$ by $q^{\alpha}(P)=P \cap \alpha$, and for $X \subseteq p_{\kappa} \lambda$ define $\nu(X)=\tau\left(\left\{\alpha<\lambda \mid q_{*}^{\alpha}(\mu)\left(X \cap p_{\kappa} \alpha\right)=1\right\}\right)$. This is a special case of the construction used by Solovay in his proof of Theorem B which we cited in the Introduction. $\nu$ is called the "glue together measure obtained from $\tau$ and $\mu$ " because we can view it as being defined by using $\tau$ to glue together the restriction measures $\mu \uparrow p_{\kappa} \alpha$ induced by $\mu$. We claim that $\nu$ is not $2^{\lambda}$-extendible. Our argument for this invokes Solovay's result that $\nu$ is a measure on $p_{\kappa} \lambda$ that does not have the partition property. This result is also available in [1, Theorem 9]. Now if $\nu$ were $2^{\lambda}$-extendible, then by Theorem 2 it would have property $\chi$, and then by Theorem $\mathrm{E}$ it would have the partition property.

\section{REFERENCES}

1. C. Di Prisco, Supercompact cardinals and a partition property, Adv. in Math. 25 (1977), 46-55.

2. T. Jech, Some combinatorial problems concerning uncountable cardinals, Ann. Math. Logic 5 (1973), $165-198$.

3. M. Magidor, There are many normal ultrafilters corresponding to a supercompact cardinal, Israel $\mathbf{J}$. Math. 9 (1971), 147-157.

4. T. K. Menas, Consistency results concerning supercompactness, Trans. Amer. Math. Soc. 223 (1976), 61-91.

5. __ A combinatorial property of $p_{k} \lambda$, J. Symbolic Logic 41 (1976), 225-234.

6. D. H. Pelletier, A condition related to the partition property for measures on supercompact cardinals, Notices Amer. Math. Soc. 26 (1979).

7. The existence of nonextendible measures on certain supercompact cardinals, Notices Amer. Math. Soc. 26 (1979), A-523.

8. The partition property for measures on $p_{\kappa} \lambda$ (manuscript).

9. F. Rowbottom, Some strong axioms of infinity incompatible with the Axiom of Constructibility, Ann. Math. Logic 3 (1971), 1-44.

10. R. Solovay, W. Reinhardt and A. Kanamori, Strong axioms of infinity and elementary embeddings, Ann. Math. Logic 13 (1978), 73-116.

Department of Mathematics, York University, Downsview, Ontario, Canada M3J 1P3 